\title{
Bilan génétique des programmes de conservation des races ovines Solognote et Mérinos précoce
}

\author{
A Djellali ${ }^{1}, \mathrm{~J}$ Vu Tien Khang $^{2}$, $\mathrm{H}$ de Rochambeau ${ }^{2}, \mathrm{E}$ Verrier ${ }^{1 *}$ \\ 1 Institut national agronomique Paris-Grignon, \\ département des sciences animales, 16, rue Claude-Bernard, \\ 75231 Paris Cedex 05; \\ 2 Institut national de la recherche agronomique, station d'amélioration génétique \\ des animaux, BP 27, 31326 Castanet-Tolosan Cedex, France
}

\begin{abstract}
Résumé - Dans le cadre des actions de sauvegarde des races domestiques menacées de disparition, des programmes de conservation génétique fondés sur une rotation des reproducteurs entre groupes ont été mis en œuvre dans les races ovines Solognote et Mérinos précoce. La présente étude a pour objet de dresser le bilan de ces programmes de conservation. L'analyse démographique montre que, conformément aux recommandations, le nombre de béliers est élevé et leur renouvellement rapide. Mais les tailles de leurs descendances ne sont pas toujours équilibrées. L'analyse génétique a été conduite à partir des données généalogiques. Les calculs de probabilités d'origine des gènes soulignent la disparité des contributions des divers élevages au stock génétique actuel, surtout en race Solognote, où le schéma rotatif repose essentiellement sur 2 élevages. Cependant, les divers fondateurs, ainsi que les divers groupes d'origine, contribuent de manière assez équilibrée au patrimoine génétique actuel. Le programme génétique empêche l'apparition de consanguinité proche et limite l'évolution de la consanguinité totale. En conclusion, les programmes de conservation génétique ont été bien appliqués, en dépit des nombreuses difficultés pratiques, et se sont révélés efficaces. C'est le déclin démographique, caractérisé par une chute rapide des effectifs et par le vieillissement des femelles, qui menace l'avenir de ces populations.
\end{abstract}

conservation génétique / mouton / analyse démographique / probabilités d'origine des gènes / consanguinité

Summary - Evaluation of the genetic conservation programmes for the sheep breeds Solognote and Mérinos précoce. In order to preserve endangered domestic breeds, genetic conservation programmes based on circular mating systems were undertaken in the sheep breeds Solognote and Mérinos précoce. The purpose of this study was to evaluate these conservation programmes. Demographic analysis indicates that both the number of

* Demande de tirés à part. 
males and their replacement rate are high in accordance with the management rules. But progeny sizes are not always balanced. Genetic analysis was carried out using pedigrees. Computation of probabilities of gene origin shows an imbalance between the contributions of different flocks to the present pool of genes, especially in the Solognote breed, where the mating system is mainly built on 2 flocks. Nevertheless, the various founder animals, as well as the reproduction groups from which they originated, contribute in a balanced way to the gene pool. The genetic programme prevents close inbreeding and restricts total inbreeding. In conclusion, the genetic conservation programmes were well implemented, in spite of practical problems, and were effective. Demographic decline, characterized by the decrease of population size and the increase of ageing, is now the main threat for the future of these populations.

genetic conservation / sheep / demographic analysis / probability of gene origin / inbreeding

\section{INTRODUCTION}

La disparition de races domestiques est un des aspects les plus spectaculaires de la réduction de la diversité génétique du cheptel exploité. Face au risque de disparition de certaines races, des actions de conservation ont été progressivement mises en place depuis la fin des années soixante. Un volet important de ces actions consiste à rechercher des débouchés spécifiques et rémunérateurs pour ces races, afin de les maintenir dans des conditions réelles d'élevage. Un autre volet vise à limiter la dérive génétique et à maintenir la variabilité de ces populations qui comptent généralement de faibles effectifs de reproducteurs. Dans cette perspective, des règles démographiques simples peuvent être proposées : un nombre élevé de reproducteurs mâles pour un nombre donné de reproductrices, un renouvellement rapide de ces reproducteurs et une uniformisation des tailles de leurs descendances (Rochambeau et Chevalet, 1990; Verrier, 1992). Des procédures plus élaborées peuvent être envisagées, comme la division de la population en groupes de reproduction et la mise en place d'un plan d'accouplement reposant sur cette notion de groupe : il s'agit des programmes dits «de rotation» qui visent à assurer une circulation des gènes dans l'ensemble de la population et à limiter ainsi la perte de certains allèles du seul fait du hasard. L'efficacité de ces méthodes de gestion génétique a été démontrée $a$ priori, par la modélisation de populations réelles et l'analyse de l'évolution attendue de certains paramètres tels que les coefficients de consanguinité et de parenté, les probabilités d'origine des gènes ou encore la distribution du nombre de copies des gènes fondateurs (Rochambeau et Chevalet, 1979; Rochambeau et al, 1979; Rochambeau et Chevalet, 1985; Chevalet et Rochambeau, 1985, 1986).

L'application de ces méthodes à des populations maintenues en ferme soulève cependant de nombreux problèmes pratiques, concernant notamment les aspects économiques et d'organisation (voir, par exemple, Verrier et Rochambeau, 1992). Aussi est-il intéressant de dresser le bilan de programmes de conservation génétique fonctionnant depuis plusieurs années, afin de vérifier s'ils ont été bien appliqués et s'ils se sont révélés efficaces. Ce type d'approche a posteriori fait appel aux méthodes de l'analyse démographique et génétique, conduite à partir des données 
d'état civil (Vu Tien Khang, 1983). De telles méthodes ont été utilisées pour décrire la structure démographique et génétique de diverses populations ovines et bovines françaises (Duraud, 1983; Avon et Vu Tien Khang, 1989; Barillet et al, 1989; Giraudeau et al, $1991 \mathrm{a}$ et b; Prod'Homme et Lauvergne, 1993). L'objet du présent article est d'établir le bilan des programmes de conservation génétique des races ovines Solognote et Mérinos précoce, qui ont été jusqu'ici bien suivies dans le cadre d'organismes professionnels structurés.

La race Solognote est une ancienne race autochtone, dont l'originalité génétique par rapport à d'autres races françaises a été établie à l'aide du polymorphisme biochimique (Nguyen, 1979). Elle fait preuve d'une remarquable sobriété et d'une grande faculté d'adaptation à d'importantes variations du disponible alimentaire au cours de l'année (Lefeuvre et al, 1980). Elle s'est toujours développée et reste aujourd'hui cantonnée dans des régions à faibles potentialités agricoles, notamment la région qui lui a donné son nom.

La race Mérinos précoce est issue du travail de sélection entrepris par des éleveurs de Champagne et du Soissonnais dans la deuxième moitié du XIX ${ }^{\mathrm{e}}$ siècle, en vue d'améliorer les aptitudes bouchères du Mérinos, introduit en France un siècle plus tôt. Cette race s'est développée dans des régions riches de grande culture, en particulier l'Est du Bassin parisien et le Chatillonnais.

Ces 2 races ont vu leurs effectifs décroître fortement dans le courant du $\mathrm{XX}^{\mathrm{e}}$ siècle. La principale cause de cette décrue réside dans la régression de l'élevage ovin dans les régions d'implantation de ces races (Hureau, 1980; Verrier, 1982). Dans le cas de la Solognote, s'est ajoutée une désaffection due à une conformation bouchère médiocre, tandis que le Mérinos précoce subissait la concurrence de races à aptitudes bouchères plus marquées, telles que l'Ile-de-France. Sous l'impulsion de leurs responsables professionnels, et avec l'aide du ministère de l'Agriculture, ces races font l'objet d'actions de conservation, depuis 1969 pour la Solognote (avec la mise en place d'un programme de gestion génétique en 1975) et depuis 1980 pour le Mérinos précoce.

Les programmes de gestion génétique de ces 2 races reposent sur les mêmes principes, mais avec des modalités d'application sensiblement différentes (Rochambeau, 1983). La race Solognote a été initialement divisée en 11 "familles», sur la base des coefficients de parenté entre animaux : dans une même famille, ont été rassemblés des animaux qui étaient plus apparentés entre eux qu'avec ceux des autres familles. Le plan d'accouplement prévoit que les mâles d'une famille donnée soient accouplés avec des femelles d'une autre famille, toujours la même, l'ordre des familles ayant été établi de façon définitive (programme dit «sans décalage»). La race Mérinos précoce, quant à elle, a été initialement divisée en 16 groupes définis indépendamment des liens de parenté entre animaux : afin de faciliter la mise en ouvre du plan d'accouplement, un groupe correspondait à un élevage ou à une fraction d'élevage. Par ailleurs, l'ordre des familles dans le plan d'accouplement change tous les 2 ou 3 ans (programme dit «avec décalage»). Dans les 2 races, les animaux, mâles et femelles, procréés dans le cadre du plan d'accouplement sont automatiquement affectés à la famille (ou au groupe) de leur mère. 


\section{MATÉRIEL ET MÉTHODES}

\section{Données}

Les données de base sont issues des fichiers raciaux, qui rassemblent des informations collectées dans les élevages par les établissements de l'élevage, les syndicats de contrôle de performances et les associations raciales. Pour chaque animal, ont été retenus les éléments suivants : numéros de l'individu, de son père et de sa mère, campagne ${ }^{1}$ de naissance, éleveur naisseur, sexe, campagne de sortie du fichier, numéro de famille (ou groupe de reproduction). Cette dernière information n'a pas été enregistrée de façon systématique au cours des dernières campagnes. Conformément aux programmes de gestion génétique, on a attribué le numéro de famille de sa mère à chaque animal dépourvu de numéro de famille, après avoir vérifié que cette procédure donnait des résultats cohérents pour les animaux à numéro de famille connu. L'objectif de la présente étude étant de faire un bilan du fonctionnement des programmes de conservation génétique, seuls ont été retenus les animaux nés depuis la mise en place de ces programmes. Les fichiers ont été épurés à la suite de divers tests de vraisemblance. En race Solognote, le fichier final rassemble 7750 animaux nés pendant les campagnes 1975 à 1991 et issus de 27 naisseurs. En race Mérinos précoce, il rassemble 10678 animaux, nés pendant les campagnes 1980 à 1992 et issus de 12 naisseurs.

\section{Analyse démographique}

Un élevage est dit actif au cours d'une campagne donnée si au moins une naissance y a été enregistrée. L'évolution du nombre d'élevages actifs au cours du temps a été observée, ainsi que la durée d'activité des différents élevages recensés. En comparant le numéro d'élevage naisseur de chaque animal enregistré avec celui de son père, on a établi une matrice dont l'élément $n_{i j}$ représente le nombre d'animaux nés dans l'élevage $i$ et issus d'un père né dans l'élevage $j$. Une telle matrice permet de décrire la circulation des reproducteurs mâles entre élevages. Un animal est dit reproducteur si l'on a enregistré dans le fichier au moins un animal dont il est le parent. L'analyse des stratégies de choix et d'utilisation des reproducteurs a concerné essentiellement les reproducteurs mâles, compte tenu de leur importance dans le fonctionnement des programmes de conservation. La distribution du nombre de reproducteurs mâles fournis par chaque élevage, sur l'ensemble de la période considérée, a été établie. Les intervalles moyens de génération ont été calculés sur les voies père-produit et mère-produit. Enfin, la distribution du nombre de descendants par reproducteur mâle a été analysée.

\section{Analyse génétique}

L'analyse génétique concerne l'ensemble des mâles actifs pendant les 2 dernières campagnes (30 béliers Solognots et 34 Mérinos précoce). L'information généalogique disponible a été décrite grâce au calcul du pourcentage d'ancêtres connus selon le

\footnotetext{
${ }^{1}$ La campagne $n$ est définie comme la période comprise entre le $1^{\text {er }}$ septembre de l'année $n-1$ et le 31 août de l'année $n$.
} 
rang d'ascendance. La diffusion des gènes dans la population a été appréhendée par le calcul des probabilités d'origine des gènes. Ce calcul quantifie le poids génétique des différentes catégories de fondateurs, en fonction notamment de l'élevage et de la famille (ou du groupe) d'origine. Enfin le calcul des coefficients de consanguinité, au sens défini par Malécot (1948), complète cette analyse. Ces coefficients dépendent de la quantité d'information généalogique considérée : aussi a-t-il été calculé en fonction du nombre de générations d'ascendants (Vu Tien Khang, 1989).

\section{RÉSULTATS}

\section{Analyse démographique}

En race Solognote, l'effectif de brebis inscrites au programme de conservation est passé d'environ 750 en 1975 (date de la mise en place du programme) à plus de 2000 en 1984. Depuis cette date, l'effectif a chuté assez rapidement : il est passé aujourd'hui en dessous de son niveau de 1975. En race Mérinos précoce, le nombre de brebis inscrites au programme de conservation a diminué assez régulièrement depuis la mise en place du programme en 1980 : il est ainsi passé d'environ 1800 à moins de 900 têtes. De manière concomitante à la chute des effectifs d'animaux au cours des dernières campagnes, on enregistre une réduction du nombre d'éleveurs adhérant aux programmes de conservation dans les 2 races (fig 1 ).

L'analyse de la durée d'activité des élevages révèle d'importantes différences entre les 2 races (fig 2 ).

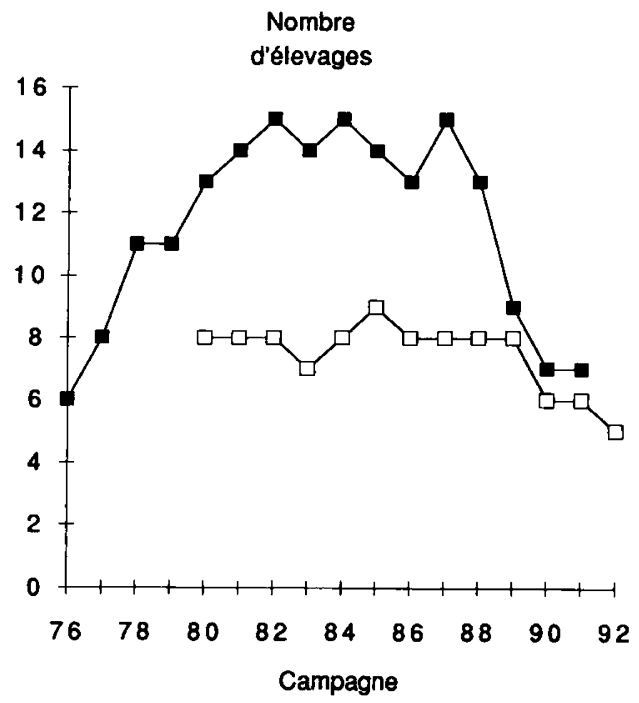

Fig 1. Évolution du nombre d'élevages participant au programme de conservation génétique dans les races ovines Solognote et Mérinos précoce; - Solognote; $\square$ Mérinos précoce.

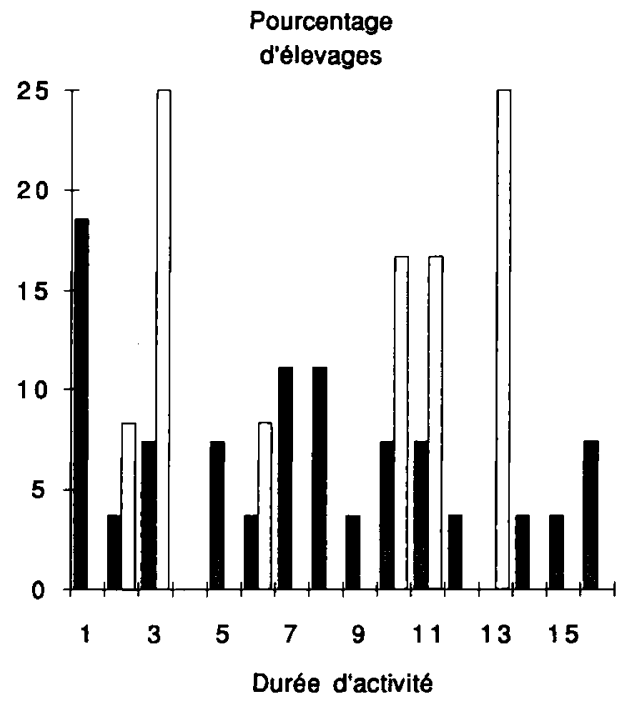

Fig 2. Distribution de la durée d'activité (années) des élevages dans les programmes de conservation génétique des races ovines Solognote et Mérinos précoce; $\square$ Solognote; $\square$ Mérinos précoce. 
En race Solognote, sur les 27 élevages ayant enregistré des naissances dans le cadre du programme de conservation entre 1975 et 1991, seuls 2 élevages ont été actifs pendant toute la période considérée, alors que 8 l'ont été pendant seulement une à 3 campagnes. La race Mérinos précoce se caractérise par une plus grande stabilité des élevages : sur un total de 12 élevages ayant enregistré des naissances entre 1980 et 1991, on compte 6 élevages ayant eu une période d'activité d'au moins 10 ans, dont 3 actifs sur l'ensemble de la période.

Les contributions des élevages en reproducteurs mâles présentent d'importantes disparités en race Solognote : ainsi, un élevage fournit presque $40 \%$ des reproducteurs et les 2 premiers élevages approchent les $60 \%$. Les contributions des divers élevages Mérinos précoce sont plus équilibrées. Hormis quelques élevages à très courte durée d'activité, les élevages des 2 races ont donné naissance à au moins un reproducteur, utilisé dans l'élevage même ou dans un autre élevage. La matrice décrivant la circulation des reproducteurs entre élevages illustre l'intensité des échanges de reproducteurs, notamment en race Mérinos précoce. Sur l'ensemble de la période considérée, un élevage Solognot a reçu des reproducteurs de 2,7 élevages extérieurs en moyenne, et a cédé des reproducteurs à un nombre équivalent d'élevages. Le chiffre correspondant est de 5,3 en Mérinos précoce. Il n'apparaît pas de stratification des élevages, telle que celle décrite par Stewart (1952), avec une hiérarchisation en éleveurs sélectionneurs et éleveurs multiplicateurs. Cependant, des différences d'organisation apparaissent entre les 2 races. En race Solognote, l'élevage détenant le plus important cheptel de brebis a eu une activité d'échange largement plus développée que celle des autres : il a cédé des reproducteurs à 18 élevages (soit les 2 tiers de tous les élevages recensés) et a reçu des reproducteurs de 14 élevages extérieurs. En race Mérinos précoce, les échanges sont plus équilibrés, avec notamment un groupe de 7 élevages parmi les 12 qui ont tous eu 2 à 2 des échanges réciproques.

Les effectifs de béliers actifs par campagne, qui étaient respectivement de 32 et de 48 dans les populations Solognote et Mérinos précoce au cours de la campagne 1982, a eu tendance à se réduire au fil du temps, pour atteindre une vingtaine d'individus lors des dernières campagnes connues. Environ $80 \%$ des mâles ont une durée de vie inférieure ou égale à 3 ans, ce qui indique une utilisation courte par rapport au potentiel biologique de l'espèce. L'évolution des intervalles entre générations confirme cette observation. Sur la voie père-produit, l'intervalle de génération est compris entre 2,5 et 3,5 ans. Sur la voie mère-produit, l'intervalle est de l'ordre de 4,5 années, avec une tendance à l'augmentation au fil du temps.

La distribution du nombre total de produits enregistrés par reproducteur mâle diffère entre les 2 races (fig 3 ). En race Mérinos précoce, c'est la classe «1 à 5 descendants» qui est la plus nombreuse (la moyenne étant de 30,8), alors qu'en race Solognote, ce sont les classes « 11 à 25 descendants» et « 26 à 50 descendants» (la moyenne étant de 22,3). Dans les 2 races, la taille de certaines descendances dépasse 150 animaux.

\section{Analyse génétique}

La figure 4 représente, pour les ensembles de reproducteurs considérés dans la présente étude et dans divers travaux antérieurs, le pourcentage d'ancêtres connus 


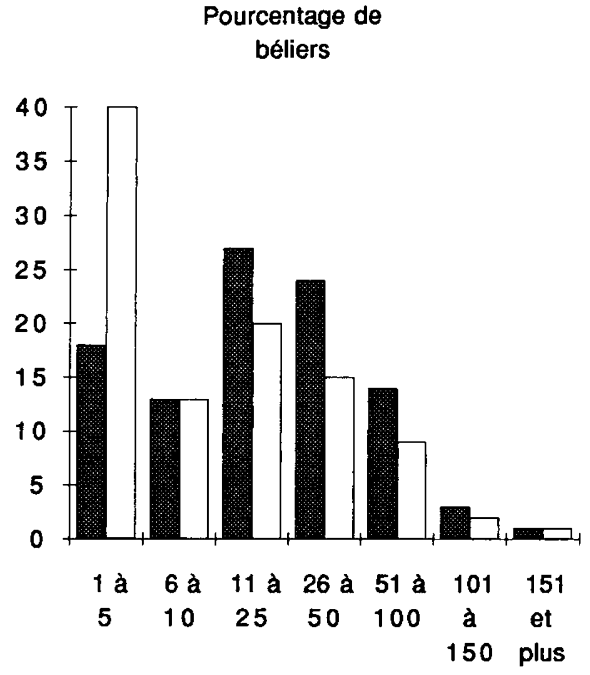

Classe de nombre de produits par bélier

Fig 3. Distribution du nombre de produits (mâles et femelles) enregistrés par bélier dans les races ovines Solognote et Mérinos précoce depuis la mise en place des programmes de conservation génétique; $\square$ Solognote; $\square$ Mérinos précoce.

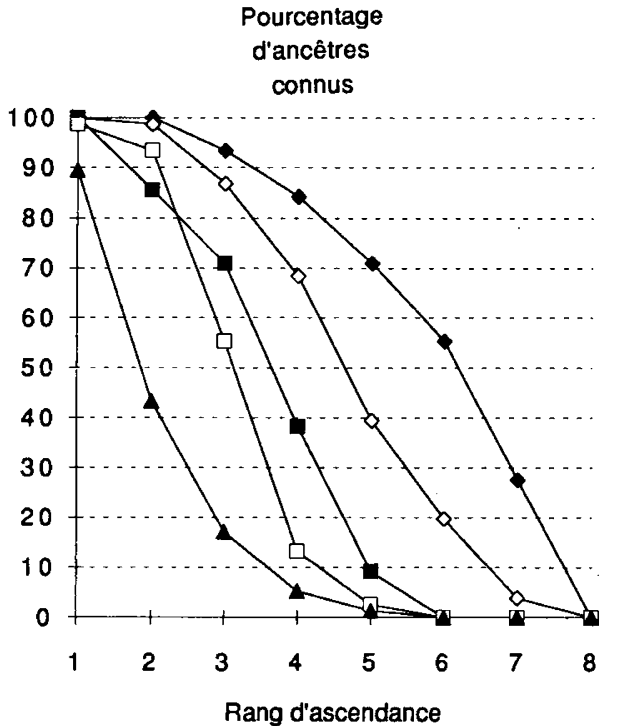

Fig 4. Pourcentage d'ancêtres connus en fonction du rang d'ascendance lors d'études généalogiques conduites dans les races ovines Lacaune (Barillet et al, 1989), Île-deFrance (Duraud, 1983), Solognote (présente étude), Mérinos précoce (présente étude) et dans la race bovine Villard-de-Lans (Avon et $\mathrm{Vu}$ Tien Khang, 1989). $\downarrow$ Lacaune; $\downarrow$ Île-de-France; $\rightarrow$ Solognote; $\square-\square$ Mérinos précoce; $\_$Villard-de-Lans.

selon le rang d'ascendance. La quantité d'information généalogique disponible est similaire dans les races Solognote et Mérinos précoce. Au-delà de la troisième génération, elle s'amenuise rapidement.

Les généalogies des mâles actifs remontent respectivement à 97 et 105 fondateurs dans les races Solognote et Mérinos précoce. La majorité de ces fondateurs est née avant la mise en place des 2 programmes de conservation. Les autres fondateurs sont des animaux entrés à titre initial dans les 2 populations. Ils proviennent le plus souvent de nouveaux élevages, mais on ne peut certifier qu'ils ne sont pas apparentés avec les fondateurs précédents.

Les courbes de contribution cumulée des élevages dont sont issus les fondateurs, classés par ordre décroissant de contribution génétique, traduisent des situations assez différentes dans les 2 races (fig 5). En race Solognote, 2 élevages contribuent pour plus de la moitié au stock génétique, mais on note ensuite un nombre assez élevé d'élevages à faibles contributions. En race Mérinos précoce, les élevages sont moins nombreux et leurs contributions plus homogènes.

Les contributions génétiques individuelles des fondateurs sont assez homogènes aussi bien en race Solognote qu'en race Mérinos précoce. Enfin, les courbes de contri- 
bution cumulée des familles ou des groupes dont sont issus les fondateurs, classés par ordre décroissant de contribution génétique, sont sensiblement rectilignes, ce qui montre que les divers familles ou groupes d'origine sont représentés de manière équilibrée dans le stock génétique actuel des 2 races (fig 6).

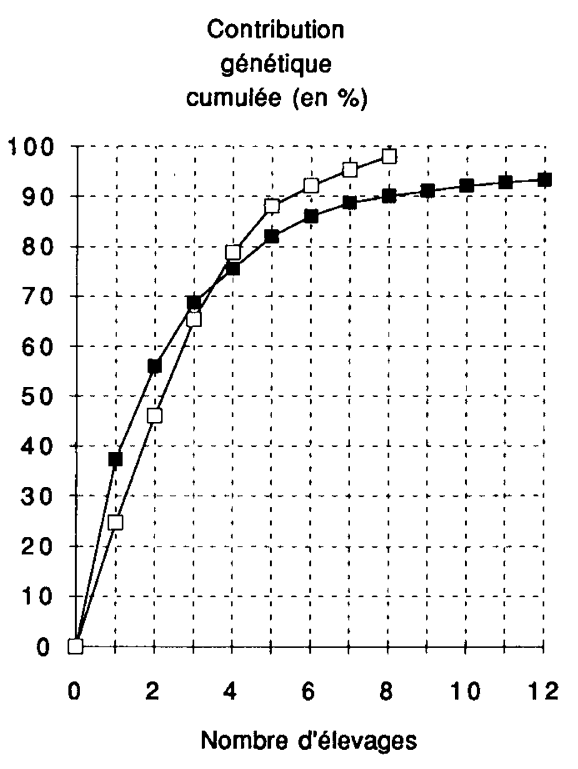

Fig 5. Contribution génétique cumulée des élevages classés en ordre de contributions décroissantes dans les races ovines Solognote et Mérinos précoce. La contribution génétique d'un élevage est définie comme la probabilité qu'un gène désigné au hasard chez un individu de l'échantillon considéré (ensemble des béliers actifs au cours des 2 dernières campagnes étudiées) soit la copie d'un gène appartenant à un fondateur issu de cet élevage; -2 - Solognote; $-\square$ - Mérinos précoce.

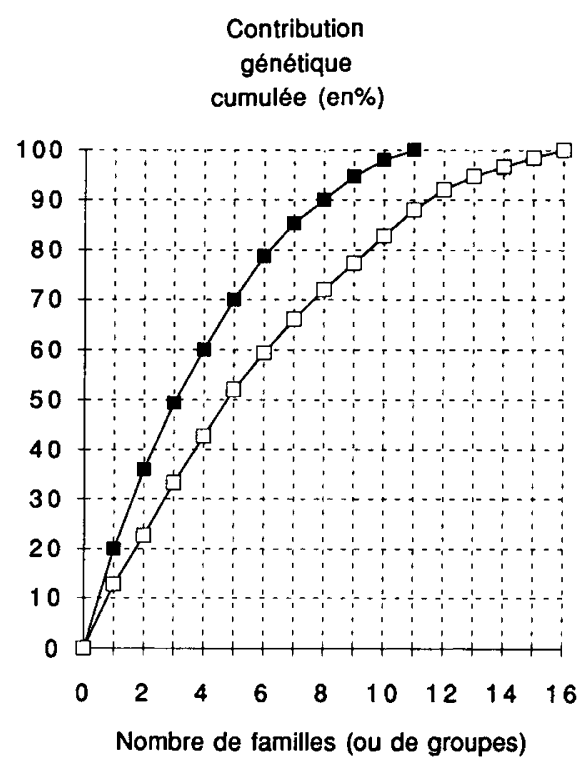

Fig 6. Contribution génétique cumulée des familles ou des groupes d'origine classés en ordre de contributions décroissantes dans les races ovines Solognote et Mérinos précoce. La contribution génétique d'une famille (ou d'un groupe) est définie comme la probabilité qu'un gène désigné au hasard chez un individu de l'échantillon considéré (ensemble des béliers actifs au cours des 2 dernières campagnes étudiées) soit la copie d'un gène appartenant à un fondateur issu de cette famille (ou de ce groupe); $-\rightarrow$ Solognote; $\square$ - Mérinos précoce.

Le coefficient moyen de consanguinité des mâles actifs est d'environ $0,5 \%$ dans les 2 races. En race Mérinos précoce, l'essentiel de la consanguinité observée est proche (les chaînes de parenté remontent à des ancêtres communs de rang inférieur ou égal à 2) et concerne moins de $10 \%$ des animaux. Cela résulte d'une dérogation accordée par les techniciens de l'UPRA à des éleveurs qui voulaient conserver certains mâles quelques années de plus. En race Solognote, il y a moins de consanguinité proche, mais plus de $30 \%$ des animaux sont consanguins. L'ensemble des résultats concernant les coefficients de consanguinité doivent être interprétés en tenant compte du caractère incomplet de l'information généalogique. 


\section{DISCUSSION ET CONCLUSION}

L'analyse démographique des 2 populations étudiées montre que les programmes de conservation génétique ont été bien appliqués, en dépit des contraintes pratiques inhérentes à la mise en œuvre de tels programmes et des conditions socio-économiques particulièrement difficiles pour les éleveurs de races ovines dont les produits rencontrent des difficultés de commercialisation. Le manque de continuité de l'activité des élevages (particulièrement critique en Solognote) et le ralentissement de cette activité créent un contexte peu favorable au déroulement de ces programmes.

Les recommandations simples concernant la gestion démographique ont généralement été respectées. Le nombre des reproducteurs mâles est assez élevé, bien qu'il présente, au cours des dernières campagnes, un fléchissement préoccupant lié au déclin démographique général des 2 populations concernées. Le renouvellement des reproducteurs mâles est rapide, comme le montre l'analyse de la durée de vie des béliers et des intervalles de génération sur la voie père-produit. En revanche, les tailles des descendances des reproducteurs mâles ne sont pas toujours équilibrées, certaines d'entre elles dépassant 150 animaux.

Les programmes dits «de rotation» organisent la circulation des reproducteurs entre élevages. Les échanges de reproducteurs sont particulièrement intenses en race Mérinos précoce, où le programme impose à l'éleveur de changer régulièrement de fournisseurs de béliers (schéma avec décalage). En race Solognote, les échanges sont moins intenses car le programme ne contient pas une telle contrainte (schéma sans décalage). En outre, le programme Solognot repose essentiellement sur 2 élevages (les plus anciens en activité et les plus importants en effectif). Certains éleveurs d'ovins Solognots hésitent à s'engager pieinement dans le programme et ne fournissent pas de béliers.

L'analyse génétique montre que les programmes ont été efficaces du point de vue de la gestion de la variabilité génétique. Les calculs de probabilités d'origine des gènes révèlent que les divers fondateurs, ainsi que les divers familles ou groupes dont ils sont originaires, sont représentés ce manière relativement équilibrée dans le patrimoine génétique actuel des 2 populations. Le calcul des coefficients de consanguinité permet de vérifier que le respect des schémas rotatifs empêche l'apparition de consanguinité proche (à quelques exceptions près en race Mérinos précoce, dues à des dérogations ponctuelles) et limite l'évolution de la consanguinité totale. Le déclin démographique, qui se traduit par la réduction conjointe du nombre d'élevages et de leurs effectifs ainsi que par le vieillissement des brebis, constitue maintenant la principale menace pour la survie de ces petites populations.

\section{REMERCIEMENTS}

Les auteurs remercient M Patrice Pinguet, président du Flock-Book Solognot et M Georges Genieys, Directeur des Flock-Books Solognot et Mérinos précoce, pour l'aide qu'ils leur ont apportée dans cette étude. Le financement de cette étude a été assuré conjointement par le ministère de l'Agriculture et de la Forêt (contrat DGER / 88-11-J) et par le département de génétique animale de l'INRA. 


\section{RÉFÉRENCES}

Avon L, Vu Tien Khang J (1989) Gestion génétique des populations: le cas de la race bovine Villard-de-Lans. In: Colloque sur la gestion des ressources génétiques des espèces animales domestiques. Paris, 18-19 avril 1989, Lavoisier, Paris, 181-190 Barillet F, Vu Tien Khang J, Roussely M et al (1989) Variabilité génétique dans le noyau de sélection des ovins laitiers de race Lacaune: une étude rétrospective. In: Colloque sur la gestion des ressources génétiques des espèces animales domestiques. Paris, 18-19 avril 1989, Lavoisier, Paris, 71-80

Chevalet C, Rochambeau H de (1985) Predicting the genetic drift in small populations. Livest Prod Sci 13, 207-218

Chevalet C, Rochambeau $\mathrm{H}$ de (1986) Variabilité génétique et contrôle des souches non consanguines. Sci Tech Anim Lab 11, 251-257

Duraud S (1983) Analyse démographique et génétique de la population ovine Îlede-France en vue d'une évaluation de son programme génétique. Mémoire de fin d'études, INA-Paris-Grignon

Giraudeau L, Casañe D, Verrier E, Rochambeau H de, Ménissier F (1991a) Analyse de la structure d'une race à petits effectifs: le cas de la race bovine Parthenaise. Elev Insémin 242, 3-14

Giraudeau L, Verrier E, Rochambeau H de et al (1991b) Survey and management of the genetic variability of a cattle breed of small population size: a successful programme in the Parthenaise breed. In: 42nd Annual Meeting of the European Association for Animal Production. Berlin, 9-12 September 1991

Hureau F (1980) Gestion génétique des petites populations ovines Chios (Grèce) et Mérinos précoce (France). Mémoire de fin d'études INA-Paris-Grignon

Lefeuvre JC, Rodde C, Clément B, Touffet J (1980) Possibilités d'élevage de moutons de race rustique dans les landes des Monts d'Arrée. 2. Étude comparée du régime alimentaire de 5 races. Bull Ecol 11, 765-773

Malécot G (1948) Les mathématiques de l'hérédité. Masson, Paris

Nguyen TC (1979) Polymorphisme sanguin du mouton et distances génétiques entre les races. In: $5^{e}$ Journées de la recherche ovine et caprine Paris, 5-6 décembre 1979, INRA-ITOVIC, 245-254

Prod'Homme P, Lauvergne JJ (1993) The Merino Rambouillet flock in the National Sheep Fold in France. Small Rumin Res 10, 303-315

Rochambeau H de (1983) Gestion génétique des populations d'effectif limité : aspects méthodologiques et application aux races d'animaux domestiques. Thèse de Docteur Ingénieur, INA-Paris-Grignon

Rochambeau $\mathrm{H}$ de, Chevalet $\mathrm{C}$ (1979) Gestion des petites populations. In : $5^{e}$ Journées de la recherche ovine et caprine Paris, 5-6 décembre 1979, INRAITOVIC, 282-292

Rochambeau H de, Chevalet C (1985) Minimisation des coefficients de consanguinité moyens des petites populations d'animaux domestiques. Génét Sél Evol 17, 459-480

Rochambeau H de, Chevalet C (1990) Genetic principles of conservation. In : Proceedings of the 4th World Congress on Genetics applied to Livestock Production. Édinbourg, 23-27 juillet 1990, vol 13, 434-442 
Rochambeau H de, Chevalet C, Malafosse A (1979) Le contrôle de la consanguinité dans les petites populations. Bull Tech Dép Génét Anim INRA 31

Stewart A (1952) The expansion and structure of the New Zealand pedigree Jersey breed. Proc N Z Soc Anim Prod 12, 32-39

Verrier E (1982) La race ovine Solognote et ses systèmes d'exploitation: histoire, situation actuelle, orientations. Mémoire de fin d'études INA-Paris-Grignon

Verrier E (1992) La gestion génétique des petites populations. INRA-Prod Anim Hors série «Éléments de génétique quantitative et application aux populations animales», 265-271

Verrier E, Rochambeau H de (1992) Problems of inbreeding in small populations and management of rare breeds. In: European Workshop on Data Collection, Conservation and Use of Animal Genetic Resources. Hanovre, 8-9 décembre 1992 $\mathrm{Vu}$ Tien Khang J (1983) Méthodes d'analyse des données démographiques et généalogiques dans les populations d'animaux domestiques. Génét Sél Evol 15, 263298

Vu Tien Khang J (1989) A. FORTRAN subroutine to compute inbreeding and kinship coefficients according to the number of ancestral generations. Comp Appl Biosci 5, 199-204 